\title{
Qualcomm Tricorder Xprize Final Round: A Review
}

Anton Gradišek, Maja Somrak, Mitja Luštrek, Matjaz Gams

Department of Intelligent Systems and Solid State Physics Department

Jozef Stefan Institute

Jamova cesta 39, 1000 Ljubljana, Slovenia

anton.gradisek@ijs.si

ABSTRACT: The Qualcomm Tricorder XPRIZE competition began in January 2012, with the goal of developing a mobile device to monitor health parameters and quickly diagnose several common medical conditions. In August 2014, a list of ten finalists was announced, including a Slovenian team MESI Simplifying diagnostics that brings together companies MESI, D.Labs, and Gigodesign, and partners from academia, Jozef Stefan Institute and Faculties of Electrotechnics and Medicine of the University of Ljubljana. In this review, we present the XPRIZE competition, we briefly look at the ten finalists and more closely at the MESI Simplifying diagnostics approach. Special attention is given to the diagnostic algorithm that was developed in order to facilitate the diagnostic process.

Keywords: Quality Communication, Communication Awards, Diagonstics Algorithm

Received: 21 June 2019, Revised 20 August 2019, Accepted 2 September 2019

DOI: $10.6025 / \mathrm{jdp} / 2019 / 9 / 4 / 101-106$

(C) 2019 DLINE. All Rights Reserved

\section{Introduction}

XPRIZE, formerly known as the X Prize Foundation, is a non-profit organization that was established in order to stimulate innovation for the benefit of humanity through incentivized competition. The challenges are "audacious, but achievable, tied to objective, measurable goals" [1]. The first prize from the foundation was the Ansari XPRIZE that offered a US\$10 million prize for the first non-government organization to launch a reusable manned spacecraft into space twice within two weeks. The prize was won by an aerospace company Scaled Composites with their SpaceShipOne [2]. In the following years, other XPRIZEs were announced, such as Google Lunar XPRIZE that focuses on launching and landing a robotic spacecraft on the Moon, with sending data back to Earth.

The Qualcomm Tricorder XPRIZE [3] was launched in January 2012. The name was inspired by the science-fiction TV series Star Trek, where "tricorder" was a device that immediately diagnosed medical conditions of the patients. The sponsor of the prize is Qualcomm, an American semiconductor company that focuses on wireless telecommunications technologies. The aim of the competition is to revolutionize the healthcare system by developing an instrument capable of measuring some key health 
parameters and diagnosing a set of common medical conditions, independent of professional health care personnel. Being able to diagnose common medical conditions at home benefits both the users by directing them to see the doctor if needed and the healthcare system itself, by reducing the costs and waiting times at medical centers. To be precise, there are two criteria in the competition: i) to continuously monitor key health metrics (blood pressure, respiratory rate, heart rate, temperature and the oxygen saturation - SpO2), and ii) to diagnose a set of 13 preselected (core set) health conditions (Anemia, Atrial Fibrillation, Chronic Obstructive Pulmonary Disease (COPD), Diabetes, Hepatitis A, Leukocytosis, Pneumonia, Otitis Media, Sleep Apnea, Stroke, Tuberculosis, Urinary Tract Infection, Absence of condition) and three other conditions from an additional set (Airborne Allergens, Cholesterol Screen, Food-borne Illness, HIV, Hypertension, Hypo- and Hyperthyroidism, Melanoma, Mononucleosis, Osteoporosis, Pertussis, Shingles, Strep Throat). Furthermore, the consumer experience represented an important component of the qualifying round evaluation criteria.

Around 300 teams from all over the world entered the competition, with 34 teams reaching the qualifying round. In August 2014, ten teams were chosen for the final round of the competition that will include testing the products on real patients during the summer of 2015. The winners of the competition will be announced in January 2016.

In this review paper, we present the ten finalists and their approaches, based on the information made public so far. Some teams unveiled several details about their products while others are more secretive. We pay special attention to the MESI Simplifying diagnostics team approach and the diagnostic algorithm.

\section{Finalist Teams}

Among ten finalists, there are four teams from the United States, two from the United Kingdom, and one from each Taiwan, Canada, India, and Slovenia. The teams are presented as on the XPRIZE website, except for MESI Simplifying diagnostics that is presented separately later on.

Aezon [4] is a team of student engineers from Johns Hopkins University in Baltimore, Maryland (US), with several partners from the industry. Their solution consists of four components, each being developed by or in partnership with a specialized company. The vital signs monitoring unit is designed to wrap around the neck, like a neck support pillow, and is being designed by a startup company Aegle. The diagnostic module is exploiting microfluidic chip technology and qPCR to test for the presence of pathogens and is being developed in partnership with Biomeme. The data is processed by a smartphone app that also uses algorithms to direct users towards relevant tests. In addition, the phone uses software for spirometry, developed by SpiroSmart. The data is stored on a cloud where an API uses big data to help turn user reported symptoms into diagnostic solutions. The team also participated in an Indiegogo campaign where they raised around 5000 US\$.

CloudDX [5] is a Canadian team, associated with the company Biosign Technologies, a manufacturer of medical devices. The vital signs unit is placed around the neck; it uses two electrodes at upper chest area to monitor ECG and an ear bud with an infrared temperature sensor to measure body temperature. An ear clip uses photoplethysmograph to monitor breathing and heart rate. Blood pressure is measured by a wrist monitor with the pulse transit time approach. The diagnostic module is designed to analyze saliva, blood, and urine. The team is working with industrial partners to consolidate multiple tests onto one multi-strip cassette. In addition, an application was developed to accept data from fitness devices and to integrate them into the system.

Danvantri [6] is a team from Chennai, India, associated with American Megatrends. The main component of their product is a handheld health monitor that features a 3-Lead ECG electrode to measure ECG signals from the finger, pulse oximeter, an infrared temperature sensor, camera, 3- axis accelerometer for monitoring physical activity and a glucometer strip attachment node. Additional devices include a wireless spirometer, neckband ECG/EEG meter, otoscope, and urine sample analyzer. The data is processed and visualized either on a smartphone or on a tablet.

DMI [7] is a team from Boston-Cambridge, Massachusetts (US), connected to the DNA Medicine Institute. They developed the rHEALTH Sensor which is a device that employs fluorescence detection optics, microfluidics, and nanostrip reagents to perform a suite of hematology, chemistry, and biomarker assays from blood. The device was developed in collaboration with NASA to monitor astronaut health.

Dynamical Biomarkers Group [8] is a team from Taiwan. Their system consists of five components: Smart Vital- Sense-Patch Journal of Data Processing Volume 9 Number 4 December 2019 
and Smart Vital-Sense-Wrist module; Smart Blood Sense module; Smart Scope module; Smart Exhaler module; and Smart Urine Sense module. The modules are connected to a smartphone app that runs algorithms based on proprietary algorithms to conduct a diagnosis.

Final Frontier Medical Devices [9] is a team from Paoli, Pennsylvania (US), connected to Basil Leaf Technologies. They are developing a device called DxtER, which relies on algorithms developed by medical experience as well as on actual patient charts. Concept art for the product indicates the device is roughly spherically shaped with integrated sensors.

Scanadu [10] is a team from Moffett Field, California (US). The team's product is called Scanadu Scout, which is a diskshaped device that contains sensors for temperature, hearth rate, and blood pressure. The disk is to be held between the thumb and index finger and placed on the forehead. The data is transferred to a smartphone and processed there. No technical specifications are known yet, neither is the approach for the diagnostic module. Scanadu ran an Indiegogo campaign from May to July 2013 and managed to raise over US\$1.6 million. The campaign has also received considerable media coverage.

SCANurse [11] is a team from London, UK. Their system consists of blood, vitals, breath, and image units. No specific information was provided on their website at the time of writing.

Zensor [12] is a team from Belfast, UK, connected to Intelesens Responsive Healthcare, a company working on non-invasive vital signs monitoring. Their prototype can detect 3-lead ECG, respiration rate, temperature, and motion. $\mathrm{SpO} 2$ sensor is being developed. To diagnose medical conditions, urine and blood analysis is included, although the details have not been made public yet.

\section{MESI Simplifying Diagnostics}

MESI Simplifying diagnostics [13] is a team from Ljubljana, Slovenia. The team consists of partners from the industry and the academia. The team is led by MESI, a startup company that specializes in development of medical devices. Their flagship product is an ankle-brachial index measuring device (ABPI MD) for the detection of peripheral arterial disease. Company D Labs is responsible for a mobile app and API while Gigodesign focuses on improving the user experience and industrial design. Partners from academia come from Jozef Stefan Institute (Department of Intelligent Systems) and two faculties of University of Ljubljana, Faculty of Electrotechnics and Faculty of Medicine. Academic partners are responsible for the development of algorithms and for expert medical knowledge. The system consists of several modules [14]. A bracelet monitors activity and three vital signs, ECG, SpO2, and temperature. A "shield" module is placed on the upper arm and consists of a wireless cuff for blood pressure measurements. It also contains a patch located on the chest to measure $\mathrm{SpO}$, temperature, ECG, respiratory rate, and activity tracking. Data obtained from the bracelet and the shield module fulfill the vital signs monitoring requirement of the competition.

The diagnosis of medical conditions is performed with the help of a smartphone app and aims to recognize all conditions from the core set, together with Hypertension, Melanoma, and Strep Throat. The user, that has already performed the vital signs measurements, indicates his concern: "I feel pain" or "I feel unwell". If "pain" is chosen, the user specifies the type of the pain on a schematic human figure (such as "chest pain"). Based on vital signs data and the type of pain/feeling unwell, the algorithm generates a list of possible symptoms that the user may experience. This list is generated to include both the symptoms that the user most probably experiences at the time and would probably want to report, and also the most relevant symptoms that would help the physician or the diagnostic method set a reliable diagnosis. Based on the chosen symptoms, the algorithm then asks for a couple of additional symptoms in order to narrow down the diagnosis and direct the user to one or more specialized modules that confirm or reject the suggested diagnosis. There are four specialized modules. A module "To see" includes a camera which is used to diagnose Melanoma and Strep Throat. Using a special camera is advantageous to using the integrated camera in a smartphone since the specifications of phone cameras may vary from a model to a model. In addition, light conditions are easier to control with a dedicated module. A module "To hear" includes microphones that are used to monitor breathing - in order to detect pulmonary diseases. This module also allows user to perform a spirometry which is used to diagnose COPD. The urine module, "Pee", performs urine analysis using test strips and a camera that reads the test results. The fourth module is called "Blood" and is intended for blood tests. In order to achieve best user experience, this module should rely on non-invasive methods, such as spectroscopy, although it is more likely that a drop of blood will be required for analysis in the final version. This module is intended for detection of diabetes and anemia. 


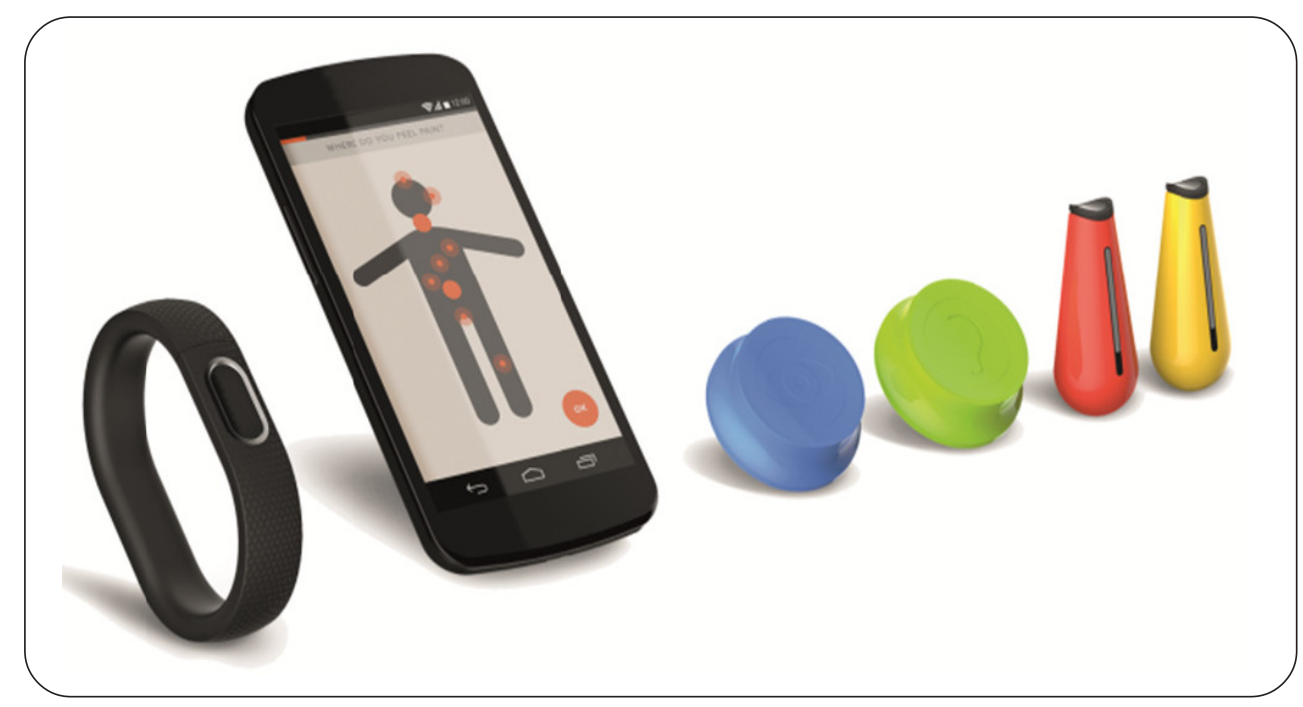

Figure 1. MESI Simplifying diagnostics system: a bracelet, smartphone app, and four diagnostic modules - To see, To hear, Blood, and Pee. The Shield module is not shown here, it comes in form of a sleeve with attachable electrodes

\section{Diagnostic Algorithm}

The diagnostic algorithm was developed at the Department of Intelligent Systems of Jozef Stefan Institute by Maja Somrak, Mitja Luštrek, Matjaz Gams, and the author of this review [15]. The aim of the algorithm is to predict the medical condition of the patient, based on the symptoms that he or she experiences. Around 60 different symptoms are taken into account. The problem is highly non-trivial. There is no simple function that would map the domain of a group of symptoms to a codomain containing a single disease. People with the same medical condition may experience different symptoms, for example, people with Otitis Media may or may not experience a headache or a discharge from the ear. An individual symptom is usually typical for several different diseases. For example, elevated temperature is typically exhibited in cases of Tuberculosis, Pneumonia, Strep Throat, Otitis Media, and others. On the other hand, even healthy people ("absence of conditions") often experience some symptoms due to reasons that are not connected to diseases. Fatigue may be related to a lack of sleep while high blood pressure may be a consequence of drinking caffeinated drinks. In addition, asking the patient for all symptoms on the list is not considered userfriendly, therefore the goal is to diagnose the medical condition as accurately as possible using as small number of questions as possible. In order to achieve the best performance, the algorithm combines expert medical knowledge and methods of artificial intelligence. At this point, we only aim to diagnose the diseases of patients with a single medical condition. Diagnosing a combination of more than one disease for a single patient is a next-level problem.

As discussed above, the initial input for the algorithm comes from the vital signs measurements (symptoms such as elevated temperature or high blood pressure) and from the pain symptoms that the user chooses. Additionally, for personalized tests, the algorithm may also include identified risk factors for a particular user (from the algorithm point of view, we also treat the risk factors as "symptoms"). For example, smokers and older people are more likely to develop COPD than non-smokers, people with a high BMI have higher risks for diabetes, etc. All these are called the "initial symptoms". The additional list of suggested symptoms is generated using association rules (ARs) and the minimum-Redundancy-Maximum-Relevance (mRMR) method. The ARs (symptom A $\rightarrow$ symptom B) are used to produce a set of probable additional symptoms. The goal of the mRMR method is to select symptoms that are as mutually dissimilar as possible and at the same time as indicative of the medical condition as possible. In other words, the algorithm tries to avoid asking the user about several similar symptoms and at the same time ask about symptoms that cover all spectrum of probable medical conditions.

In the following step, the information gathered up to this point is used for actual disease prediction. The probabilities for the 15 medical conditions are evaluated using a set of J48 classifiers, one for each of the conditions. There are two probability thresholds: conditions above the high threshold are considered very probable and conditions below the low threshold are considered unlikely. The area between the two thresholds is a so-called "gray zone" where we do not have enough information to make a reliable claim whether the medical condition is present or not. The diagnostic procedure terminates when all conditions

104 Journal of Data Processing Volume 9 Number 4 December 2019


from the list are either above the high or below the low threshold. If one or more condition remain in the gray zone, at least one additional question (symptom) is required for a confident prediction. The additional symptom is chosen according to the highest information gain (IG) that an individual symptom would bring.

Calculation of the IG and mRMR values, searching for ARs, and building the J48 classifiers is based on two types of data - real and simulated patient data. Real patient data was collected either with both patients with medical conditions and healthy individuals filling in a questionnaire about the symptoms they experience (a complete set of symptoms), or by medical doctors retroactively filling in the symptom tables for real patients. The simulated dataset was build using expert medical knowledge. Physicians prepared a table of probabilities for patients suffering from each of the medical conditions to exhibit each of the symptoms from the list, based on their professional experiences. Using this table, it is possible to generate millions of distinct "virtual patients". Initial tests using only simulated data showed high sensitivity and specificity for disease diagnostics [15]. Tests using a combination of real and simulated data are currently underway.

\section{Conclusions}

We present an overview of the Qualcomm Tricorder XPRIZE competition and the teams that reached the final round, with a special focus on the Slovenian team entry. The approaches of many teams are similar to some degree. The most common approach is to use of single a device with a number of integrated sensors to monitor vital signs (the first competition task). The second task, the diagnosis of medical conditions, is typically achieved using a series of dedicated additional modules. Some teams rely strongly on detection of biomarkers in body fluids while others also incorporate technologies such as spirometry and image-processing algorithms. Several teams mention they use algorithms for diagnostics, although not much has been revealed to the public so far.

The MESI Simplifying diagnostics approach consists of a bracelet and a "Shield" module to monitor vital signs. The diagnosis of medical conditions is obtained using an algorithm that runs on a mobile device. The algorithm uses the vital signs data and the symptoms entered by the patient to predict a possible medical condition and to direct the patient to use a specialized module which confirms or rejects the prediction.

An overview of the algorithm, developed at Jozef Stefan Institute, is presented. The algorithm combines expert medical knowledge with methods of artificial intelligence and machine-learning. The aim of the algorithm is to make an accurate prediction of diagnosis with a small number of questions, to improve the user experience. We outline the challenges of the task. Testing of the algorithm on real patient data is currently underway and the results will be published later.

\section{References}

[1] http://www.xprize.org/

[2] http://space.xprize.org/ansari-x-prize

[3] http://www.qualcommtricorderxprize.org/

[4] http://www.aezonhealth.com/

[5] http://www.clouddx.com/

[6] http://www.vitalsplus.com/

[7] http://www.dnamedinstitute.com/

[8] http://dbg.ncu.edu.tw/

[9] http://www.basilleaftech.com/

[10] https://www.scanadu.com/

[11] http://www.scanurse.com

[12] http://www.intelesens.com

[13] http://www.simplifyingdiagnostics.com/ 
[14] Somrak, M., Luštrek, M., Sušteri, J., Krivc, T., Mlinar, A., Travnik, T., Stepan, L., Mavsar, M., Gams, M. (2014). Tricorder: Consumer Medical Device for Discovering Common Medical Conditions, Informatica 38, 81-88.

[15] Somrak, M., Gradišek, A., Luštrek, M., Mlinar, A., Sok, M., Gams, M. Medical diagnostics based on combination of sensor and user-provided data. AIAM/ NetMed 2014, Artificial Intelligence and Assistive Medicine: In: Proceedings of the 3rd International Workshop on Artificial Intelligence and Assistive Medicine co-located with the 21st European Conference on Artificial Intelligence (ECAI 2014), Prague, Czech Republic, 36-40. 